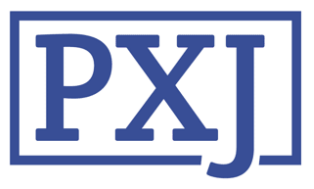

Patient Experience Journal

Volume 6

Issue 2 Special Issue: The Role of Technology

and Innovation in Patient Experience

Article 11

2019

\title{
A next-day, brief e-survey overcomes the excessive variability seen in CAHPS-style emergency department surveys so that individual physician performance can be assessed on a regular basis
}

Tom Scaletta

Edward Elmhurst Health

Eva Hare

Kellstadt Graduate College of Business of DePaul University

Christopher Sung Lee

Kellstadt Graduate College of Business of DePaul University

Follow this and additional works at: https://pxjournal.org/journal

Part of the Business Analytics Commons, Business Intelligence Commons, Emergency Medicine Commons, Health and Medical Administration Commons, Health Information Technology Commons, Health Policy Commons, Health Services Administration Commons, Health Services Research Commons, and the Technology and Innovation Commons

\section{Recommended Citation}

Scaletta T, Hare E, Lee CS. A next-day, brief e-survey overcomes the excessive variability seen in CAHPSstyle emergency department surveys so that individual physician performance can be assessed on a regular basis. Patient Experience Journal. 2019; 6(2):59-65. doi: 10.35680/2372-0247.1376.

This Research is brought to you for free and open access by Patient Experience Journal. It has been accepted for inclusion in Patient Experience Journal by an authorized editor of Patient Experience Journal. 


\section{A next-day, brief e-survey overcomes the excessive variability seen in CAHPS- style emergency department surveys so that individual physician performance can be assessed on a regular basis}

\section{Cover Page Footnote}

The authors of this work thank Andrew Gallan, PhD (Director, Department of Marketing, Florida Atlantic University) who brought together the authors as part of his DePaul graduate class on PX. We are also grateful to Julie Danker, LCSW, CPXP (Chief Experience Officer, SmartER) who provided comments on the manuscript. The views expressed in this article are those of the author(s) and not those of the Edward Elmhurst Health (Naperville, IL) or the Kellstadt Graduate College of Business of DePaul University (Chicago, IL). This article is associated with the Innovation \& Technology lens of The Beryl Institute Experience Framework. (http://bit.ly/ExperienceFramework). You can access other resources related to this lens including additional PXJ articles here: http://bit.ly/PX_InnovTech 


\title{
A next-day, brief e-survey overcomes the excessive variability seen in CAHPS-style emergency department surveys so that individual physician performance can be assessed on a regular basis \\ Tom Scaletta, Edward Elmhurst Health.tom.scaletta@eehealth.org \\ Eva Hare, Kellstadt Graduate College of Business ofDePaul University, elpfian@yahoo.com \\ Christopher Sung Lee, Kellstadt Graduate College of Business of DePaul University, c.sung.lee@gmail.com
}

\begin{abstract}
Traditional CAHPS-style emergency department (ED) surveys result in excessive variability when assessing individual physician performance. The objective of this study is to measure the variability of a brief, electronic survey (e-survey). The study team also measured the association of individual physicians to demographic data, physician and patient factors, and a physician burnout assessment tool. Data from SmartContact (SmartER, La Grange, IL) is a next-day, esurvey that takes about 30-seconds to complete. This tool was used by a hospital-employed emergency department (ED) group during calendar year 2017 across 2 EDs and 37 physicians. ${ }^{1,2}$ Variability was estimated regarding raw patient experience (PX) scores and top box scores by using intraclass correlation coefficients (ICCs). Pearson correlations were used to measure the interaction between PX scores, physician factors, and patient factors. Analysis of the 2017 calendar year showed statistically significant differences between physician PX performance on a bimonthly and quarterly basis. As well, there was lower PX in patients presenting at night. No correlation was found with a burnout assessment tool. This study demonstrates statistically valid performance differences among physicians using a next-day e-survey, which conforms to the recommendations of ED professional organizations for use in driving provider PX improvement, enhancing patient trust, and improving patient outcomes.
\end{abstract}

\section{Keywords}

CAHPS, survey, patient experience, statistical validity, variability, patient satisfaction, Press Ganey

\section{Background}

As healthcare reimbursement practices shift toward valuebased care and payments are increasingly tied to PX scores, hospitals are increasingly using these scores as key metrics to evaluate performance for both the organization and, when available, for individual providers. In some cases, these scores may be used in bonus calculations, coaching mandates, and physician remediation.

Most hospitals use data from the Consumer Assessment of Healthcare Providers and Systems (CAHPS) surveys to evaluate their programs and staff with regards to PX. ${ }^{3}$ Centers for Medicare \& Medicaid Services (CMS) has expanded CAHPS to ambulatory healthcare settings (e.g., CG-CAHPS for office practices) though their emergency department survey remains in development and has been since 2012.

A position statement from the American Academy of Emergency Medicine (AAEM) and a policy from the American College of Emergency Physicians (ACEP) require statistically-valid $\mathrm{PX}$ surveys if they are used to suggest differences in individual physician performance. ${ }^{4,5}$
Thus, it makes sense to measure physicians on a monthly, bimonthly, or quarterly basis so that they can be trended in a timely basis. Doing so allows the objective measurement of behavior change (e.g., effect of a coaching program) resulting in PX score improvement.

Edward-Elmhurst Health (EEH) is a 3-hospital healthcare system located in the western suburbs of Chicago. In 2004, EEH initiated ED callbacks by utilizing dedicated staff to gather patient-reported wellbeing and service feedback. ${ }^{6}$ In 2014, EEH leveraged technology by implementing an esurvey in its EDs. The transition from callbacks to an esurvey reduced the cost of post-discharge contact by over $80 \%$ and has been termed 'the evolution of callbacks. ${ }^{7}$ Success in reaching patients electronically is facilitated by the ubiquity of smartphones across all sociodemographic characteristics and by an increasing societal adoption of asynchronous communication. ${ }^{8}$

The SmartContact e-survey consists of 5 questions, which are sent via text message and email to all ED patients discharge home on the following morning. The e-survey takes, on average, 30 seconds to complete and allows patients to provide quantitative and qualitative feedback. 
The system allows ED staff to respond to medical issues or service complaints. EEH analyzes its e-survey data to gain insights into the performance of individual ED providers, to determine which factors correlate with high/low provider scores and to develop initiatives to improve the PX.

With CAHPS-style surveys, data on individual performance has been insufficient, and no meaningful conclusion can be drawn on physician performance. In fact, one-third of providers swing over 20 percentile points in their individual provider score from month-to-month. ${ }^{9}$ This is frustrating for practicing physicians and their medical directors.

The hospital survey vendor with greatest market share, Press Ganey (PG) (South Bend, IN), has traditionally delivered a CAHPS-style, mailed ED survey. These have a very low $(2 \%)$ response rate though this does meet the CMS minimum requirement for outpatient settings. ${ }^{10,11}$ The marked variability of quarterly physician scores using this method is demonstrated by the fact that achieving the 5 th versus 50 th percentile can equate to only 3 points on a 100-point scale.

The EEH CAHPS survey completion rate for the emergency department is $1.5 \%$. Since EEH fulltime emergency physicians are contracted to work 1600 clinical hours per year, it takes 15 months to achieve the 30 CAHPS survey minimum required to determine if an individual's performance is classified as high or low. This is an inadequate time interval to measure behavior changes and influence employment decisions.

With low sample size and high variability in ED ratings, traditional PG surveying does not conform to AAEM and ACEP standards since a minimum of 30 surveys per provider should be completed for statistical validity in order to evaluate physician performance. ${ }^{12,13}$ PG now offers an email version of the survey with a much higher sampling rate. However, the survey includes 35-questions, raising survey fatigue concerns. Also, it is not available via the preferred e-survey gateway, text message, and does not address wellbeing issues so misses risk mitigation opportunities.

An area of interest to EEH is determining whether physician PX scores can be used as an early indicator for physician burnout. Burnout is identified by its 3 key components: emotional exhaustion, depersonalization, and diminished feelings of personal accomplishment in a worksetting. ${ }^{14}$ Patients of physicians with high-exhaustion and high-depersonalization have significantly lower satisfaction scores compared with patients of physicians with lowexhaustion and low-depersonalization in a primary care setting. ${ }^{15}$
The premise that improving PX improves patient outcomes has been demonstrated in the literature. A review of 55 published PX studies demonstrated positive associations between PX and medical care quality. ${ }^{16}$ The two most frequent associations were patient-reported wellbeing and adherence to the medical recommendations. Additionally, PX for inpatient physicians was associated with patient complaints and risk management issues. ${ }^{17} \mathrm{It}$ is believed that establishing trust during an ED visit increases the likelihood that discharged patient will adhere to the medical plan. ${ }^{18}$

\section{Materials and Methods}

This study was deemed exempt by the EEH institutional review board. EEH has 3 associated EDs and 2 were included in this study. The Edward Hospital ED is a 70,000 annual volume, comprehensive community hospital located in Naperville, IL. The Plainfield ED is a 30,000 annual volume freestanding ED located in Plainfield, IL.

For this study, a SmartContact dataset was used that included all 2017 ED patient e-survey responses (23,468 surveys). It also included two datasets from Edward Hospital, which were 2017 patient visit information (104,066 records) and physician scores on a voluntary, selfadministered Professional Quality of Life Scale (ProQOL) assessment tool (27 records). ${ }^{19}$ In order to join the esurvey responses and patient visit information, a patient encounter ID was generated using patient arrival date, arrival time and date of birth, fields which appeared in both datasets. This ID was non-unique for 11 records due to inadvertent duplicate registrations and dropped from the analysis. Physician burnout scores were joined using the existing physician ID variable.

All patients discharged home from the ED during the study period received an e-survey by email or text message. Some patients reported only a "home phone number" and were reached as this number was a cell phone.

The e-surveys were not sent to those patients admitted, transferred, or who left without being seen. Additionally, patients with an ED case management plan (comprised mostly of those with opiate use disorder) were not surveyed. Finally, patients with no disposition code recorded at the time the data was cut were not surveyed.

Of the 104,066 patients included in the dataset, 77,573 were eligible to receive an e-survey based on their discharge disposition and lack of an ED case management plan. Of eligible patients, $30.3 \%$ completed e-surveys resulting in an average of 634 surveys per physician annually or 53 per physician per month. This number is well above the published boundary of 30 to achieve statistical validity for monthly benchmarking. 
The e-survey data included four versions of the question sets: English and Spanish translations and whether the respondent was the patient or the respondent's child was the patient. A total of 231 (1\%) Spanish e-surveys were completed. The survey question of interest for this analysis rates the doctor's level of concern for the patient's wellbeing (Figure 1). A similar question regarding the nurse was used for comparison. There are 5 possible survey responses which were converted to a 5-point scale: 1-Very Low, 2-Low, 3-Average, 4-High and 5-Very High. These values were also categorized based on whether the respondent gave the top box score of 5-Very High. There were comments given on 4,775 (20.3\%) of the e-surveys conducted in English.

The study assessed if the monthly rating for each physician was statistically different using ICC analysis as recommended in Pines (2017). This excluded 7 physicians who did not work for 12 consecutive months, which removed 2,552 patient e-survey responses or $10.8 \%$ of all received e-surveys. ICC analysis also did not include the 231 respondents who completed the e-survey in Spanish.

\section{Figure 1.}

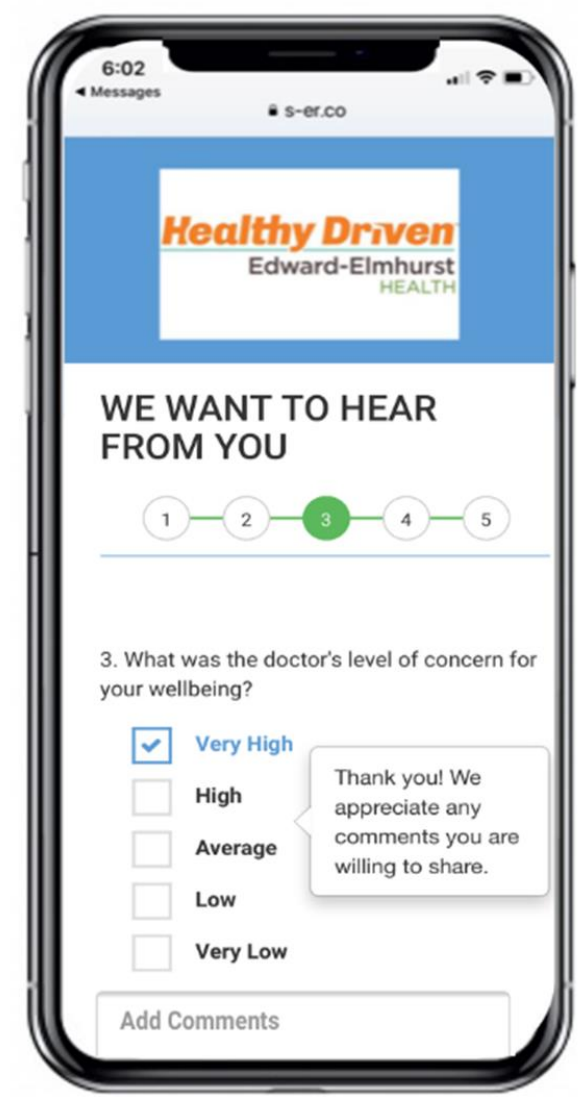

A correlation analysis was conducted to compare physician ratings to the following factors:

- Hour of arrival

- Hour of arrival by day (7A-3P), evening (3P-11P), and night (11P-7A) shift

- Arrival day of week

- Arrival day by weekday versus weekend

- Physician burnout as measured by the ProQOL 5 Assessment Tool

Operational, provider, facility, visit, and patient variables were studied with conceptual relationships to PX scores and regression analysis was used to assess construct validity. At the facility level, this included annual visit volume, proportion of physician-hours that were $11 \mathrm{PM}$ to 7 AM, median discharged length of stay, proportion of patients discharged, and the emergency severity index, which reflect acuity at the point of triage.

As a point of comparison, the correlation between nurse ratings and arrival time and date was calculated. Each correlation was computed separately and included all patients with data values for each pair of variables.

There were comments shared by patients on 4,775 (20.3\%) of the e-surveys conducted in English.

\section{Study Design and Setting}

This was a retrospective cross-sectional study using data from a next-day, 30-second electronic survey (e-survey) tool used by a hospital-employed ED group during calendar year 2017 across 2 EDs and 37 physicians. Variability regarding raw PX scores and top box scores and through intraclass correlation coefficients (ICCs) was estimated. Pearson correlations were used to measure the interaction between satisfaction scores, physician factors, and patient factors.

Operational, patient, and physician data used for the study were generated by data from the Epic EMR as well as ProQOL 5 assessment data obtained by the medical director. PX data were collected by SmartContact (SmartER, La Grange, IL). Data from these sources were combined by a research team from Kellstadt Graduate College of Business of DePaul University (Chicago, IL) at the visit level into a single database for research purposes.

\section{Results}

\section{ICC Analysis}

ICC analysis is commonly used to examine if a quantitative measurement varying within a single group is statistically significantly different. It is standard practice to consider ICC coefficients above 0.7 to show a moderate difference while a coefficient of 0.9 is often used for clinical studies 
Figure 2. Variation in Physician Monthly Score

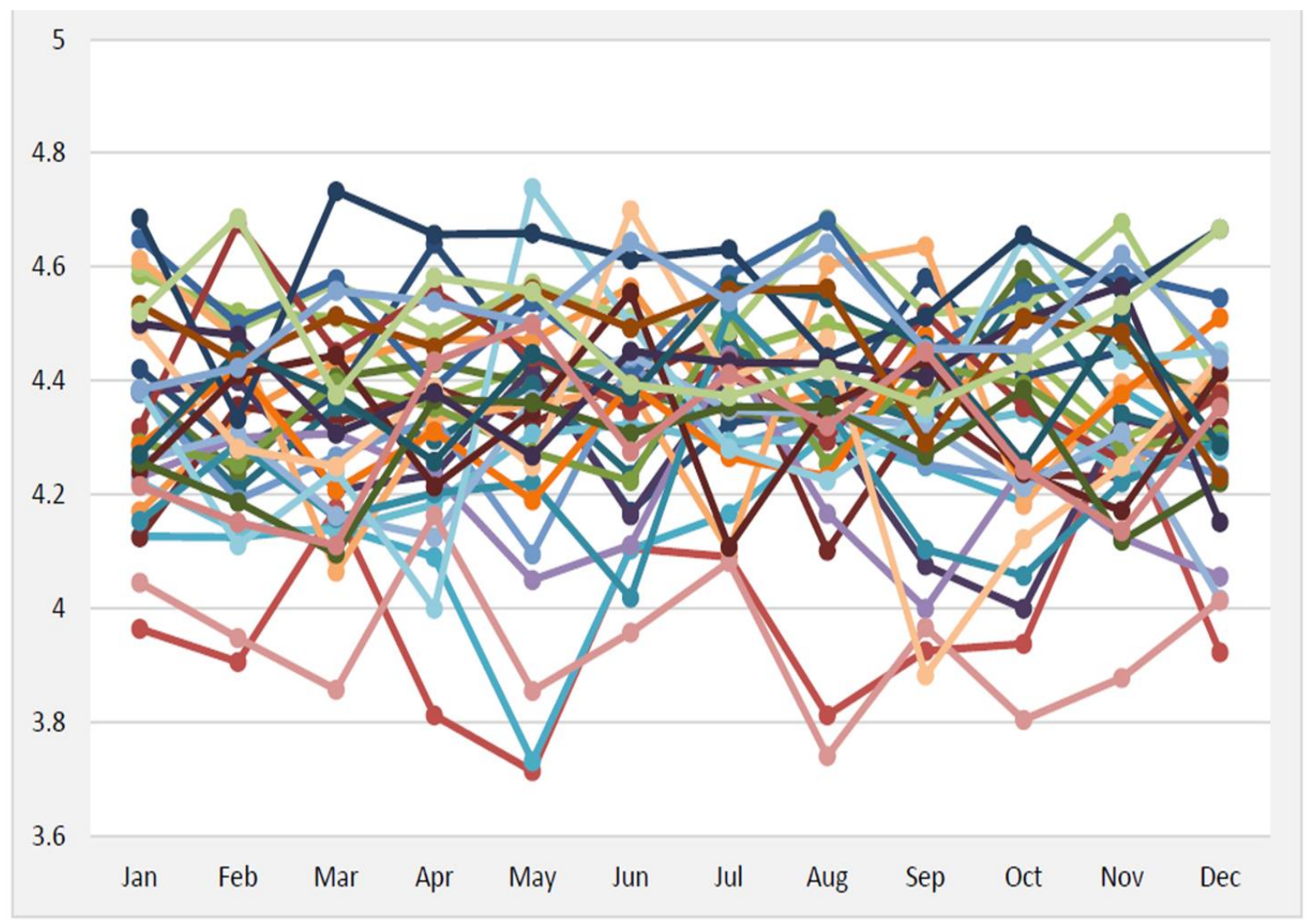

that require a strongly differentiated result. ICC was calculated using monthly average rating, percent of top box scores and a modified net promoter score (mNPS). These did not produce satisfactory results as the ICC for top box was 0.48 and the ICC for the mNPS was 0.38 . The mNPS was calculated as the number of "Very High" ratings minus ratings of "Low" or "Very Low".

It was determined that there is relatively large variation in average score for each physician at the monthly level (Figure 2) with an ICC of 0.55 and so a satisfactory coefficient score was not achieved for a monthly time period. Therefore, data was aggregated into 2-month (bimonthly) and 3-month (quarterly) time periods with ICCs of 0.70 and 0.81 , respectively. At the aggregate level, the variation decreased, and the ICC improved significantly. Because our dataset compromised only 2017 data, there were only 4 data points for each physician at the quarterly level (Figure 3).

Figures 2 and 3 visually illustrate that differences in individual provider performance becomes clear when the interval changes from monthly to quarterly.
The ICC analysis indicates that, at the monthly level, there is insufficient evidence to demonstrate a consistent difference in PX performance between physicians. However, there is enough separation between the top and bottom performers to infer a statistical difference between these groups. At the bimonthly and quarterly level, there was definite statistical evidence that some ED physicians receive consistently higher PX score than others. This finding indicates that the ED PX is impacted by which physician treats that patient.

\section{Correlation Analysis}

The Pearson Correlation was used to measure the interaction between patient ratings and various factors. The analysis showed no correlations $(<0.1)$ existed between day of week and physician and nurse ratings. Also, there was no correlation to the weekend/weekday categories $(<0.1)$, indicating arrival day does not contribute to patient rating. Of note, EEH increases emergency physician coverage on Mondays.

For time of arrival, there was no correlations $(<0.1)$ for individual arrival hours. Arrival times aggregated into day, evening, and night shifts were analyzed. Statistically 
Figure 3. Variation in Physician Quarterly Score

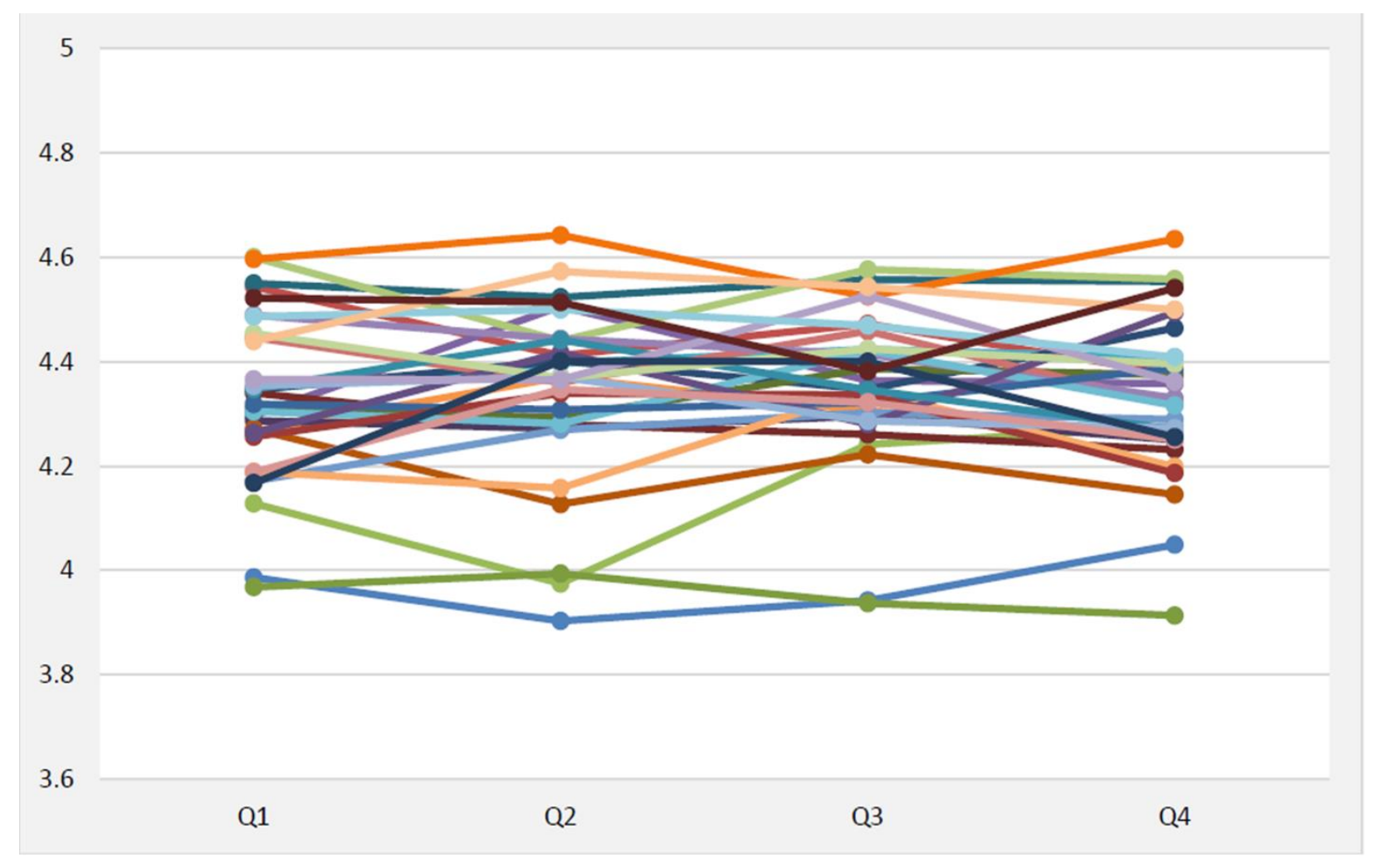

significant correlations were not achieved, but visual inspection did show a decrease in rating on the night shift for both physicians and nurses (Figure 4/5). While a 0.1 rating decrease is numerically small, our standard deviation for physician rating is 0.86 and for nurse rating is 0.78 , so even small rating variations can have substantial impact.

\section{Conclusion}

Practice implications for these findings have to do with the marked difference between next-day e-surveys and CAHPS-style surveys regarding patient participation and actionable results.

CAHPS surveys are lengthy and contribute to survey fatigue. Also, there is a long delay in obtaining patient feedback prohibiting wellbeing checks and service recovery. Therefore, many health systems limit the number of CAPHS surveys sent to the amount that assures achieving the CMS minimum requirement and are now utilizing next-day e-surveys as a means of engaging patients after an outpatient encounter.

This study demonstrates statistically valid performance differences among physicians using a next-day e-survey and conforms to the recommendations of ED professional organizations when the data is used to manage providers and drive PX improvement initiatives.

\section{References}

1. 2017: Scaletta T, Rising K, Ranney M. Automated Discharge Follow-Up Program in Value and Quality Innovations in Acute and Emergency Care (pp. 170-177) by Pines, J, Wiler J, Ward $M$ (Eds.) Cambridge University Press.

2. SmartContact 2019. accessed 4/28/19. https:// smarter.net/smartcontact/

3. AHRQ. Frequently Asked Questions about CAHPS. Agency for Healthcare Research and Quality. Accessed 3/12/2018. https://www.ahrq.gov/cahps/faq/index.html

4. AAEM Position Statement on PX Surveys in the ED 2006. accessed 4/29/19.

https://www.aaem.org/resources/statements/positio $\mathrm{n} /$ position-statement-on-patient-satisfaction-surveysin-the-emergency-department

5. ACEP Policy, Patient experience of care survey tools should be based on a statistically valid sample size free from selection bias 2016. accessed 4/29/19. https://www.acep.org/patient-care/policystatements/patient-experience-of-care-surveys/ 
Figure 4. Difference from Mean Rating by Shift

\section{2}

\section{1}

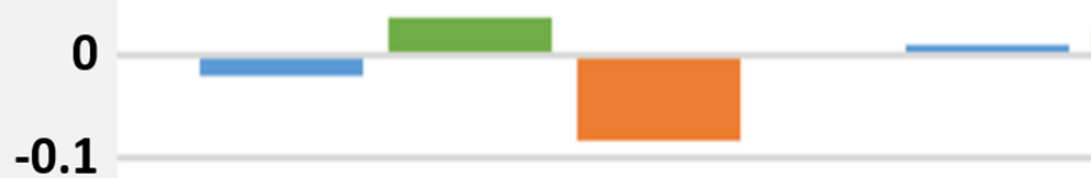

$-0.2$

\section{Physician Rating}

\section{Day}

Evening

\section{Nurse Rating}

\section{Night}

During our analysis by hour of admission, it was noticed that a disproportionately small number of e-survey responses for patients who arrived during the 23:00 hour due to a higher number of patients with an unknown discharge disposition as data was extracted about an hour after.

\section{Figure 5. Effect of Arrival Hour on Missing Discharge Dispositions}

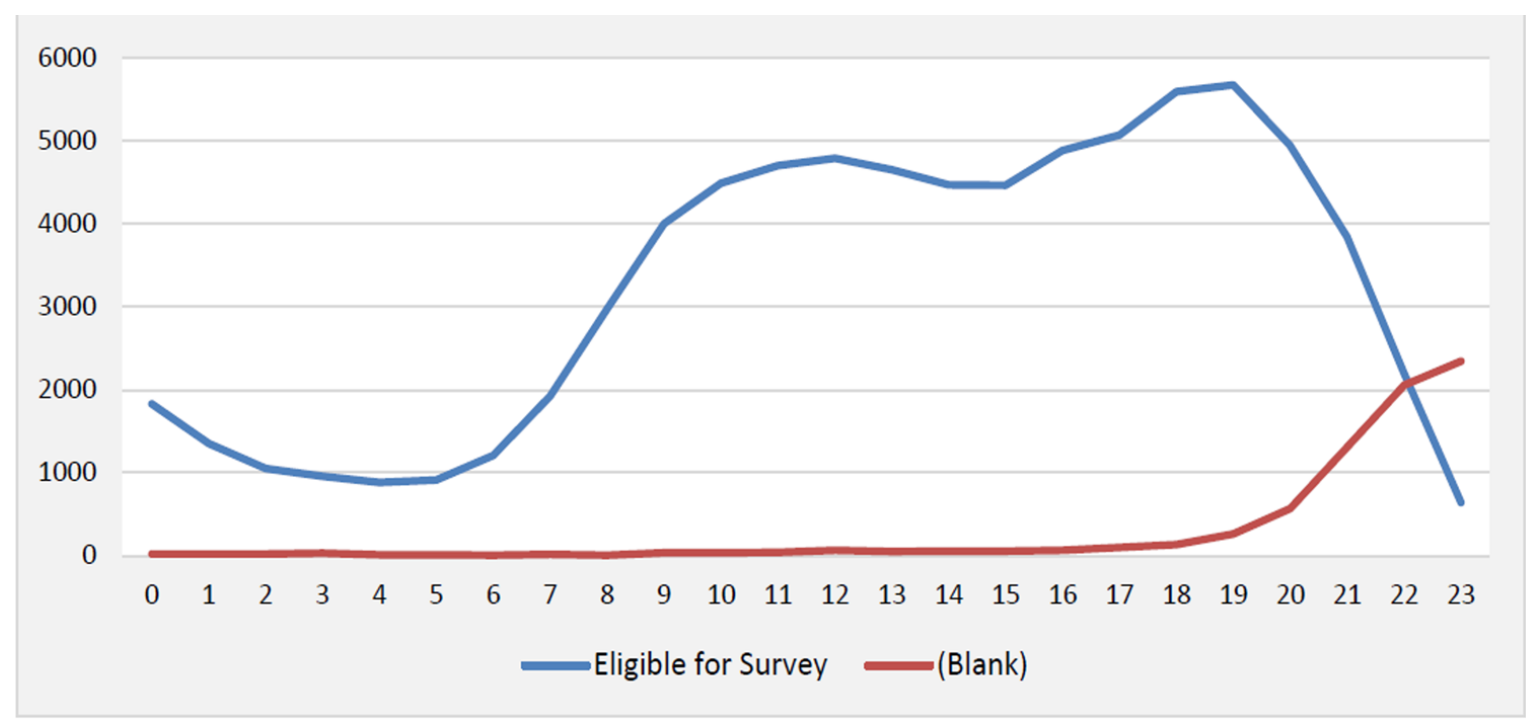

A correlation analysis of physician rating was conducted against the scores from the ProQOL 5 burnout assessment tool administered to the physicians and no correlation was found.

6. Scaletta T. Urgent Matters Best Practices: Edward ED Callback Clerk. 2005, Robert Wood Johnson Foundation. https://smhs.gwu.edu/urgentmatters/sites/urgentma tters/files/CallBackClerk.EdwardHosp.pdf

7. 2015: Papa A, Lefton C. Discharge Texting: The Evolution of ED Callbacks. Journal of Emergency Nursing July;41(4):345-

346. http://www.jenonline.org/article/S00991767(15)00128-2/abstract
8. Smartphone pentration to reach $68 \%$ in 2018. Mobile Advertising Forecasts 2017.Zenith.

https://www.zenithmedia.com/smartphonepenetration-reach-66-2018/

9. Pines J, Pennint P, Alfaraj S et al. Measurement Under the Microscope: High Variability and Limited Construct Validity in Emergency Department PatientExperience Scores. Ann Emerg Med 2018, 71(5):545554. https://doi.org/10.1016/j.annemergmed.2017.11.011 
10. Handel DA, French LK, Nichol J, Momberger J,

Rongwei F. Associations between Patient and

Emergency Department Operational Characteristics

and Patient Satisfaction Scores in an Adult

Population. Ann Emerg Med 2014, 64(6):604-

8 http:/ /www.annemergmed.com/article/S0196-

0644(14)01114-7/abstract

11. Gupta M. Happy Meals for Everyone? Ann Emerg Med. 2014;64(6):609-611.

https://doi.org/10.1016/j.annemergmed.2014.09.023

12. Hogg and Tanis' Probability and Statistical Inference (7e). 2017.

13. ACEP 2017 Information paper-Statistically-valid

Patient Satisfaction Surveys. Accessed 4/29/19.

https://www.acep.org/globalassets/uploads/uploade d-files/acep/clinical-and-practice-

management/policy-statements/informationpapers/empc-statistically-valid-patient-satis.surveys_bd-ltr_draft-10172017-mm.pdf

14. Sameer S, Chopra A, Wayne M et al. Sotile, M. Physician Burnout. JAMA 2004. 291(5), 633.

15. Anagnostopoulos, F., Liolios, E., Persefonis, G. et al. Physician Burnout and Patient Satisfaction with Consultation in Primary Health Care Settings: Evidence of Relationships from a one-with-many Design. J Clin Psychol Med Settings 2012. 19:40

16. Doyle C, Lennox L, Bell D. A systematic review of evidence on the links between patient experience and clinical safety and effectiveness. BMJ Open 2013. 3:e001570. https://bmjopen.bmj.com/content/bmjopen/3/1/e0 01570.full.pdf

17. Stelfox H, Gandhi T, Orav E, Gustafson M. The relation of patient satisfaction with complaints against physicians and malpractice lawsuits. Am J Med 2005. 118(10):1126-1133. http://www.ncbi.nlm.nih.gov/pubmed/16194644

18. Scaletta T. Achieving Great Outcomes in Your Discharged ED Patients. Common Sense (AAEM). 2019. https://www.aaem.org/UserFiles/file/MAAEMSerie sJanFeb19.pdf

19. Professional Quality of Life (ProQOL 5). Access 4/29/19. https://proqol.org/uploads/ProQOL_5_English.pdf 